\title{
ORAID: An Intelligent and Fault-Tolerant Object Storage Device*
}

\author{
Dan Feng**, Lingfang Zeng, Fang Wang, and Shunda Zhang \\ Key Laboratory of Data Storage System, Ministry of Education, \\ School of Computer, Huazhong University of Science and Technology, Wuhan, China \\ dfeng@hust.edu.cn, zenglingfangatom.com
}

\begin{abstract}
Hints for traditional storage system come from three aspects: file or directory attribute layout, user input and file content analysis. But in the OBS (object-based storage), object, a new fundamental storage component different from traditional storage unit (file or block), provides ample hints for storage system, which are help for designing more intelligent (or smarter) storage device. RAID (redundant arrays of independent disks) is a popular mechanism to offer fault-tolerance storage. But RAID based on file or block interface has very limited intelligence. This paper presents a novel object-based device: ORAID (object RAID). ORAID consolidates disk space of individual disk into a single storage spool and implements object-sharing and fault-tolerance through the object-interface. In ORAID, storage object is stored in form of original block fragments and their XOR verification fragments are among the disks of the device. As the higher abstract of data encapsulation, storage object provides more intelligent properties, and ORAID has more effective ability to implement online data re-layout and online capacity expansion.
\end{abstract}

\section{Introduction}

With exponentially increasing information, storage research community is quite aware of the management problem of storage system. Enhancing storage device intelligence, specially self-managing and self-configuring, has been well-publicized calls to action. Examples include the recovery oriented computing, the Self-* Storage [1], University of California Santa Barbara's Active disks [2], CMU Active disks [3]. University of California at Berkeley's IDISK [4] offloads application-specific functions, such as database scanning, in order to reduce server processing load. SDDS [5] discusses mechanisms for detecting and recovering from non-recoverable read errors and drive failures, introducing improved techniques for detecting errors in disk reads and fast

* This work was supported by the National Basic Research Program of China (973 Program) under Grant No. 2004CB318201, Huo Yingdong Education Foundation under Grant No.91068, the National Science Foundation of China under Grant No.60273074 and No.60303032.

** Corresponding author. 
recovery from disk failure. ISTORE [6] achieves self-maintenance via hardware platform with integrated diagnostic support, reactive self-maintenance by a layered, policy-driven runtime system that provides a framework for monitoring and reaction, proactive self-maintenance with support for continuous on-line self-testing and component characterization and a standard API for interfacing applications to the runtime system. OceanStore [8] is a global persistent data store designed to scale to billions of users. It is designed to have a very long MTTDL, but at the cost of dramatically increasing the number of disk requests per block written.

Above mentioned are typical smart storage device. Local intelligence is achieved in those devices and is a basis of the whole storage system. In addition, RAID [9], which has been developing for approximately twenty years, shows itself in some forms: software RAID [20], defined as a system that distributes data redundantly across an array of disks attached to each of the workstations connected on a high-speed network, provides higher throughput and availability. HP AutORAID [7] is attribute-managed storage and a two-level storage hierarchy implemented inside a single disk array controller. Reference [10] has proposed iSCSI RAID (or iRAID for short) to improve iSCSI performance from the iSCSI target point of view. iRAID provides a direct and immediate solution to boost iSCSI performance and improve reliability. Specially, ORAID, as an intelligent and fault-tolerant object storage device, is introduced in this paper.

The rest of this paper is organized as follows: Section 2 describes the background and our motivation. Section 3 studies those characteristics of storage object, such as object attribute, object method and object-based storage policy. Section 4 provides the implementation and typical application of ORAID. And the conclusions and the future works are given in section 5.

\section{Background and Motivation}

Indeed, in the past 20 years, the use of technology improvements to achieve lower cost, as well as increased performance, has been a major theme in the storage industry. For data storage, OBS (object-based storage) is the next wave of storage technology and devices [13]. Smart storage system is very important for emerging data-intensive network applications. An entire industry and research community has emerged to achieve fault-tolerance and smarter storage by many ways: (1) Modifying file system.

(2) Smarting storage device. (3) Enhancing network fabric intelligence. (4) Considering overall storage system. All of these endeavors have been productive, but they cannot help industry to bridge the gap between intelligence storage system and real business value when they get insufficient hints from file or block.

Security and performance of aggregate bandwidth are emphasized in NASD project [12] and Lustre cluster file system [16], respectively. The function of RAID is mainly realized by data layout policy of the metadata server, the same to the iRAID.

It may be a new approach for us to design smart and fault-tolerance storage device with the help of object storage. This principle has been considered for many years in the storage system community. But traditional storage systems based on files or blocks, such as DAS, NAS and SAN, cannot provide more accurate or ampler hints. But, objects in OBS already give useful hints to the storage system, in the form of object 
OID and other attributes, and that storage system can successfully get smarter from these hints. Also, with those hints, RAID-based storage device may change its RAID running level (online re-layout works) or online capacity expansion. Hints about an object's access pattern, size, and lifespan can aid in a variety of ways including improving the object's layout on RAID and increasing the effectiveness of managing and caching [17].

\section{Overview of Storage Object}

Objects are of variable size and can be used to store entire data structures, such as files, database tables, medical images, or multimedia. Object data is application data and include some attributes (size, create time, object type, lifespan, ownership etc.) [11], [12], [15], [16]. The object is the fundamental unit of data storage in OBS. Also, object is a logical collection of bytes with well-known methods for access, attributes describing characteristics of the data, and some policies that realize self-management.

\subsection{Storage Object Attribute Is the Foundation Stone of Hints}

Object represents an abstract higher-level. A storage object is variable-length and can be used to store any type of data. Thus different type of data has different attributes. If we use a uniform style to contain attributes for any type of object, more disk space may be wasted while saving objects with their attributes in disks. So we propose a scalable way to present attribute, which we call attribute card. The attributes in the same attribute card have close relationship. An attribute card can be identified by an attribute card ID (AID), and an attribute within the attribute card is indexed by an attribute index (AIdx), so an attribute is uniquely presented as a pair (AID, AIdx).

We define a common attribute card, which contains attributes that every object possesses. For example, any object has attributes such as create time, size, last modified time et al, all of which can be included in the common attribute card. Any new type of attribute card can be defined according to applications, and such attribute card is application-defined attribute card. Considering attributes of a multimedia object, a multimedia attribute card can be defined as follow: QoS, frame rate, video size et al. However, a database record attribute card may be composed of attributes as follow: record count, record format et al.

In OBS, ORAID, an object-based storage device, is also a special device object and comprises some attributes, such as ORAID's initial capacity, remaining capacity and IP address. For convenient managing, other object attribute can be extended to three types, such as public attribute, privacy attribute, share attribute. Share attribute (we want to make a difference between access attribute and access pattern) contains information about its environment, group and user access control information. E.g., share attribute provides greater interoperable access to objects and provide information to ORAID that describes the meaning of the objects and facilitates more effective data management, such as on-disk layout and resource provisioning. Privacy attribute (similar to storage attribute [4]) can tell the storage system how to deal with an object, for instance the level of ORAID to apply, the size of the capacity quota or the performance parameters 
required for that data. Once ORAID accesses to attributes describing the objects, it can use these attributes to achieve improvements in the device. E.g., ORAID might make sure that the hot spot object is available more quickly, or that previous versions of objects are automatically saved when it detects that objects are being changed. When ORAID understands the meaning of data it contains, it can use that information to create new storage applications that take advantage of ORAID ample processing power. In addition, information such as backup or QoS (quality of service) requirements, object access patterns (sequential or random), or relationships to other objects, is also stored as share attributes.

Traditional systems can automatically learn to classify the properties of files and predict the properties of new files by exploiting the strong associations between a file's properties and the names and attributes assigned to it [18], [19]. ORAID analyses object pattern from the following three aspects: (1) Object attribute analysis, (2) Access-based object attribute analysis (including application assistance and existing user input), (3) Inter-object relationships. However, we have to emphasize that not only can ORAID get those hints (most of them are local), but also metadata server can attain some hints from global object metadata information.

\subsection{Storage Object Method Is the Executive Unit}

Traditionally, a block-based disk can only perform READ and WRITE operations on data. Object-based device changes this way by allowing users to upload application-specific operations at storage device and performing operations on data while receiving requests from clients. Furthermore, object-based device must provide more flexible and scalable operations and can be applied to more application fields. OBS provides high management by extending object method.

Each object in an ORAID also has associated with read/write streams. Users can insert any modular methods into read and write streams. Within a stream, methods are connected one to the next to form a method chain. When users read/write objects, the objects enter the stream at one end, progress through the method chain, and exit at the other end. By the form of method chain, object method is executed when the data passing through the chain. ORAID provides flexible methods by supporting arbitrary data stream operations. Thus, users can upload any kind of methods, and build any kind of method chains for any objects.

For ORAID, users can associate an object with specific method chains in two ways: transient way and persistent way. In transient way, method chains can be built when an object is opened and destroyed when the object is closed. In persistent way, method chains for an object can be created or destroyed through a register/un-register request to ORAID. After an object is associated with its method chains through registration, the operations in the method chain are always implicitly performed.

\subsection{Storage Object Policy Is the Brains of ORAID}

Object storage policy management is rule-based scripts that identify ORAID conditions, states, and events, and generate appropriate actions. In the network storage world, it easily tracks and acts upon object storage network devices (e.g. ORAID) and 
infrastructures. Because of the scalability of object-based storage, policy developers are easy-pressed to code policies that can run in object storage environments. There are so many storage realities to observe, such as runaway storage data, greedy database applications that ruthlessly grab disk space. Within this demanding environment, OBS use policies to help them meet service-level agreements (SLAs), keeping object storage devices provisioned and configured so that they can meet required space, performance, and availability metrics.

SNIA [22] defines storage-related policies as "the measurable, enforceable, and realizable specification of methods, action and/or desired states that meet service requirements in a storage-based information infrastructure." This means that policies are machine-based reactions that deploy in response to changes in the object storage environment. These changes might be driven by events, conditions, or computing processes. Users can measure and enforce both changes and policy-generated reactions from a user interface.

Object storage policies may not be as mature as network storage policies, but they are important additions to the storage management thoughts. Object storage policy can manage object storage devices to improve and automate backup-and-restore and archiving procedures, supply bandwidth to demanding applications, and assure that a critical backup has the resources it needs.

In our ORAID, rules and policies are separated. It is because that one policy may correspond to several rules, and one rule may adapt to different policies. The rule pool is filled with general values regarded to be useful by one or more of the management policies. Most popular rules such as time, recency of access [15], frequency of access, capacity and size are initially registered to the pool. A new policy registered into the policy pool has to contact corresponding rule in case they are not already registered. So ORAID can provide a solution for dynamic loading or unloading policy.

Also, for ORAID, those policies themselves are just descriptions of how to help OBS implement system management function and specify system local states and how to response to them. These may include any proposed storage system management policy because some state information may be exchange among ORAIDs. When clients or application services operate objects, metadata server (has global information) and ORAID (has local information) set correlative object attribute values. By getting object attributes and statistic values, OBS will ensure that the relative policy for the current workload or performance is triggered ${ }^{1}$ (in ORAID) and therefore have the largest effect on the storage system management decisions.

\section{Design, Implementation and Application of ORAID}

\subsection{Functions of ORAID and Its Metadata Server}

Figure 1(a) shows those function modules in ORAID. With SCSI subsystem and RAID driver, the lower disk array realizes those functions of traditional hardware RAID.

${ }^{1}$ OBS triggers the policy depending on the compare between object attribute values and rules from the rule pool. 
Moreover, in the middle layer of figure 1(a), the storage layout frame provides online re-layout and online capacity expansion. In fact, the function of online re-layout includes the RAID level management (or configure). All above mentioned implementations all require the support of logic volume management which is realized by the logical volume manager (LVM) [21]. The logic volume management is used for online disk storage management. The logic volume management considers all installed disks as pools of data storage and provides easy-to-use online disk storage management for computing environments.

With those online self-managing functions, the management of ORAID does not require that machines be taken off-line at a major convenience to users. So, in the distributed client/server environment, databases and other resources maintain high availability in ORAID. ORAID is easy to access, improves performance and ensures data availability and integrity. However, if we want ORAID to carry out those function without manual intervention, different from traditional RAID, ORAID have to attain some intelligence. Fortunately, with those help of object interface in figure 1(a) and object storage service in figure 1(b), storage objects in ORAID can present adequate hints. Figure 1 also shows that metadata server and ORAID provide storage services for clients (or users) by high-speed TCP/IP network.

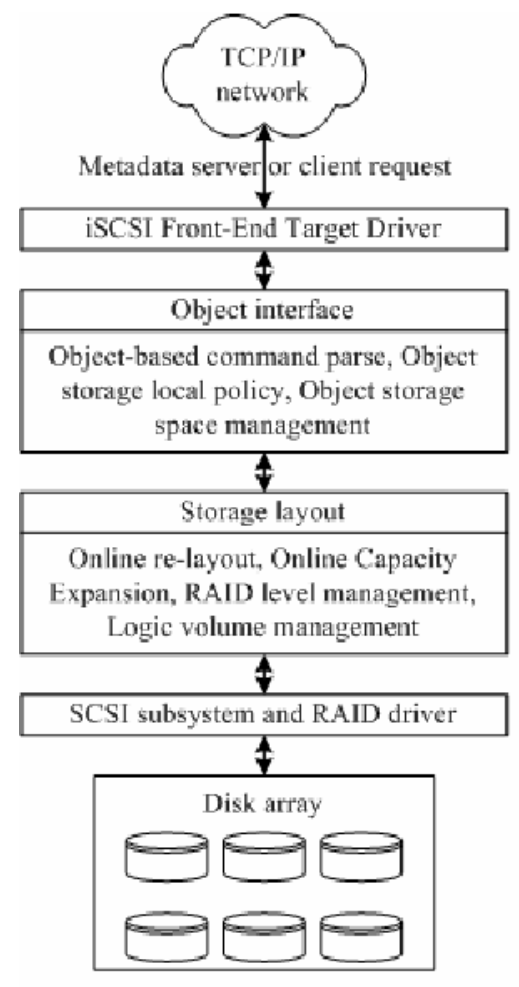

(a) ORAID

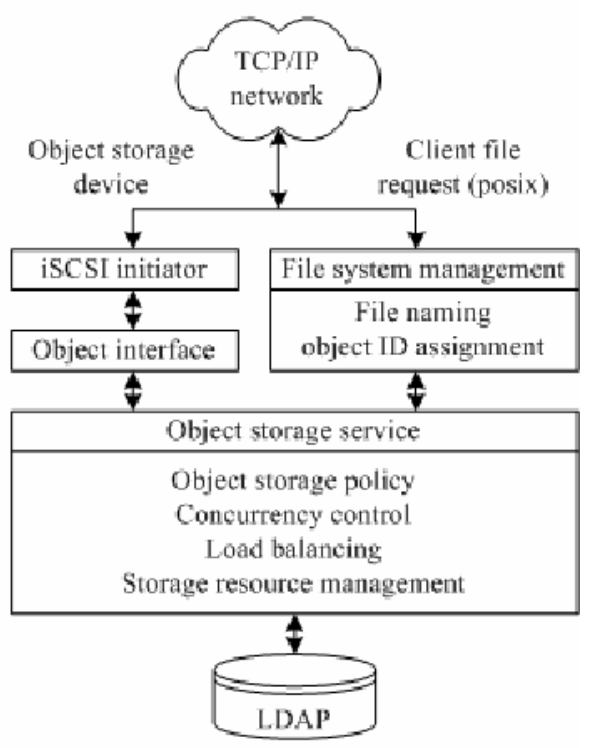

(b) Metadata server

Fig. 1. Function modules of the ORAID and the metadata server 


\subsection{Program Implementation of Metadata Server}

Flow chart of program in metadata server is showed in figure 2. In metadata server, most of those requests are write/read service of clients (or users). Other services of clients include permission, rm, mkdir, ls etc. In fact, the security of object access also is very important, but, it is not the key objective of this paper. Figure 2 does not show the service of object storage policy. In the following section 4.3, we will discuss this together with storage local policy in ORAID device.

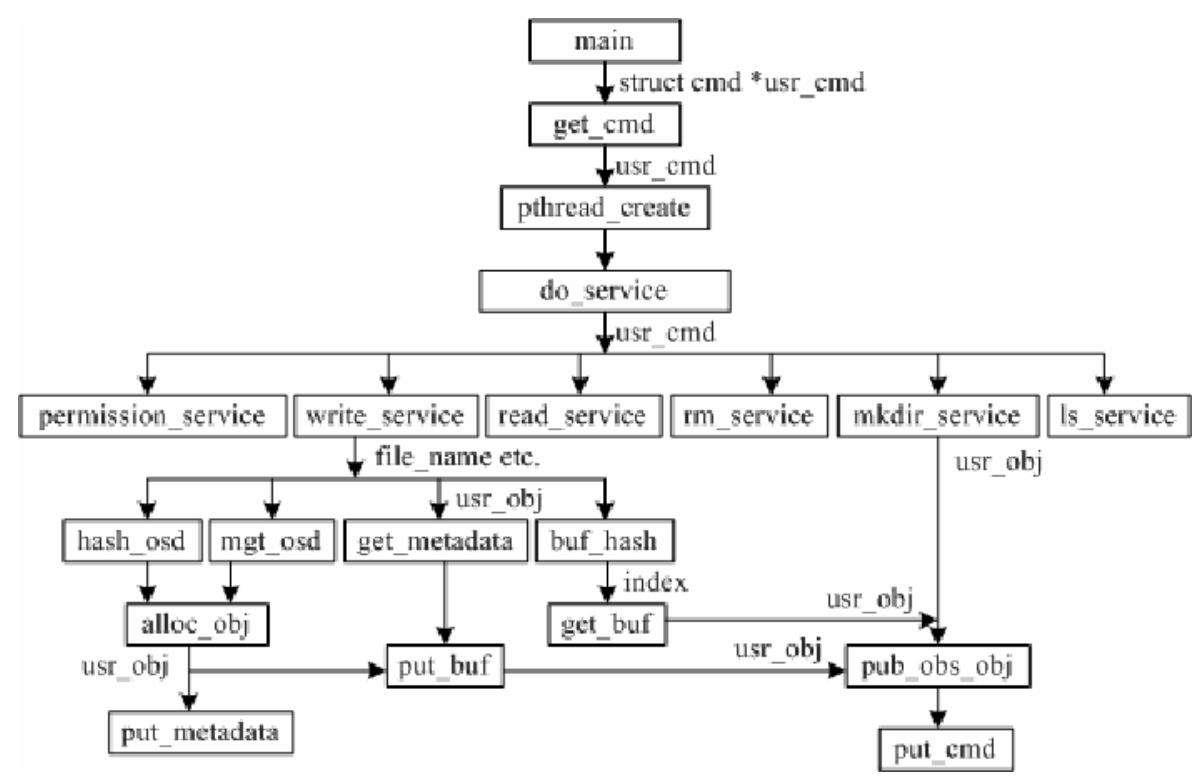

Fig. 2. Flow chart of program in metadata server

\subsection{Implementation of Object Command and Local Policy in ORAID}

As an object storage device, ORAID must implement object-based storage device commands [23]. Figure 3(a) provides the parse program for object-based command in our ORAID, and thes implementation is based on Intel Lab's software reference implementation of iSCSI [24].

The processing flow of storage object policy is showed in figure 3(b). In ORAID, object and policy are operated by application services or resource management. Besides some system methods, object also has some itself methods. Obviously, object attributes are set some values, or storage subsystem can get some statistic information by object attributes (Of course, both ORAID and metadata sever record some access information when objects are operated by application services.). By comparing rules, some policies may be triggered in ORAID and some adaptive code in mechanism library may be executed according to those policies. 
With those hints provided by storage objects, ORAID usually changes the storage layout to change the redundancy or performance characteristics of the storage. The logic volume management adds redundancy to storage either by duplicating the address space (e.g. mirroring) or by adding parity (e.g. RAID-5). Performance characteristics of storage can be changed by changing the striping parameters which are the number of disks and the stripe width.

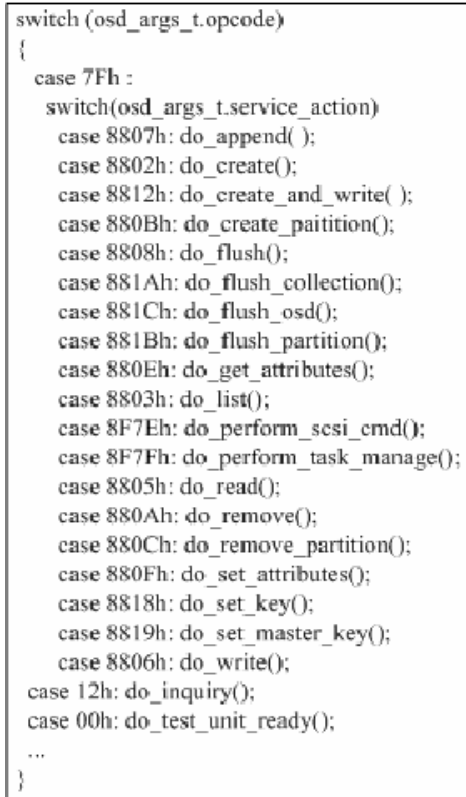

(b) Storage object policy

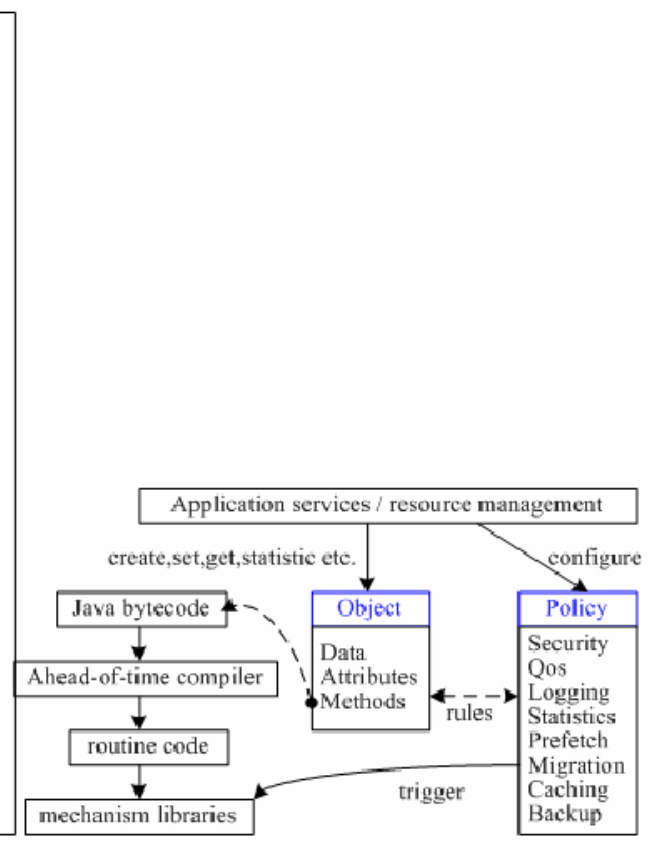

Fig. 3. Implementation of object-based command and storage object policy in ORAID

ORAID makes changing fault-tolerant storage configuration easy, without requiring a data backup and restore cycle. If ORAID composes of hot-pluggable disks (e.g. SATA disk), storage expansion or extension can be performed online -- without shutting down the server operating system. Online capacity expansion adds storage capacity to an array. If an existing array is nearly full of data, simply by adding a new physical disk, ORAID can expand the capacity without disturbing the existing data. According to those storage object policies, ORAID automatically sets the disk hardware and configuration.

In addition, in object-based storage system, above mentioned works are cooperatively finished between ORAID and metadata server. For instance, ORAID must send some state message to metadata server when it is configuring its layout. Also, metadata server must record capacity expansion of ORAID. 


\section{Conclusions}

As business trends evolve and new technologies emerge, storage system is becoming more complex, compounding the challenge of sustaining the intelligence that storage community seeks. This paper has shown that object attributes are strong hints of how that object will be accessed and managed in a smart object-based storage system. Then storage object method and policy are introduced. Furthermore, a novel intelligent and distributed fault-tolerance storage mechanism called ORAID is provided. Using those characteristics of storage object in OBS, ORAID realizes fault-tolerance storage, online re-layout and online capacity expansion.

\section{References}

1. John D. Strunk, and Gregory R. Ganger. A Human Organization Analogy for Self-* Systems. First Workshop on Algorithms and Architectures for Self-Managing Systems, in conjunction with Federated Computing Research Conference (FCRC). San Diego, CA, June 2003.

2. A.Acharya, M.Uysal, and J.Saltz. Active disks: programming model, algorithms and evaluation. Proceedings of the 8th Conference on Architectural Support for Programming Languages and Operating System (ASPLOS VIII), pp. 81-91, Oct. 1998.

3. E. Riedel, C. Faloutsos, G.A. Gibson, and D. Nagle. Active disks for large-scale data processing. Computer, Vol 34, Issue: 6, pp. 68-74, June 2001.

4. K.Keeton, D.A.Patterson, and J.M.Hellerstein. A case for intelligent disks (IDISKs). SIGMOD Record, 27(3), Sept. 1998, available at http://www.acm.org/sigmod/record

5. Qin Xin, E.L.Miller, T. Schwarz, D.D.E. Long, etc. Reliability Mechanisms for Very Large Storage Systems. Proceedings of 20th IEEE/11th NASA Goddard Conference on Mass Storage Systems and Technologies, pp. 146-156, April 2003.

6. A.Brown, D. Oppenheimer, K. Keeton, R. Thomas, J. Kubiatowicz, and D.A. Patterson. ISTORE: Introspective Storage for Data-Intensive Network Services. Proceedings of the 7th Workshop on Hot Topics in Operating Systems (HotOS-VII), Rio Rico, Arizona, March 1999.

7. John Wilkes, Richard Golding, Carl Staelin, and Tim Sullivan. The HP AutORAID Hierarchical Storage System. In High Performance Mass Storage and Parallel I/O: Technologies and Applications, IEEE Computer Society Press and Wiley, pp. 90-106, 2001.

8. Chris Wells. The OceanStore Archive: Goals, Structures, and Self-Repair. March 2004, available at http://oceanstore.cs.berkeley.edu/publications/index.html

9. D.A. Patterson, et al. A Case for Redundant Arrays of Inexpensive Disks (RAID). ACM International Conference on Management of Data (SIGMOD), pp: 109-116, 1988. QUID Web Proxy Cache, available at http://www.squid-cache.org

10. Xubin He, Praveen Beedanagari and Dan Zhou. Performance Evaluation of Distributed iSCSI RAID. International Workshop on Storage Network Architecture and Parallel I/Os. New Orleans. September 2003.

11. Butler W. Lampson. Hints for Computer System Design. In ACM Operating Systems Review, volume 15(5), pp. 33-48, October 1983.

12. Thomas E. Anderson, Michael D. Dahlin, Jeanna M. Neefe, David A. Patterson, Drew S. Roselli, and Randolph Y. Wang. Serverless network file systems. ACM Transactions on Computer Systems (TOCS), Vol 14, Issue: 1, pp. 41-79, February 1996.

13. P. J. Braam. The Lustre storage architecture. Technical report, Cluster File Systems, Inc., January 2004, available at http://www.lustre.org/docs/lustre.pdf 
14. SNIA, Object-Based Storage Devices (OSD) workgroup, January 2004, accessible from http://www.snia.org/osd

15. M. Mesnier, G.R. Ganger, and E. Riedel. Object-based storage. Communications Magazine, IEEE, Vol 41, Issue: 8, pp. 84-90, Aug. 2003.

16. Intel Corporation. Object-Based Storage: The Next Wave of Storage Technology and Devices. January 2004, accessible from http://www.intel.com/labs/storage/osd/

17. Ke Zhou, Jiang-Ling Zhang, Dan Feng, and Zhi-Kun Wan. Cache prefetching adaptive policy based on access pattern. Proceedings of the First International Conference on Machine Learning and Cybernetics, Beijing, Vol 1, pp. 496-500, Nov. 2002.

18. Michael Mesnier, Eno Thereska, Daniel Ellard, Gregory R. Ganger, and Margo Seltzer. File Classification in Self-* Storage Systems. Proceedings of the First International Conference on Autonomic Computing (ICAC-04), New York, May 2004.

19. Craig A.N. Soules, and Greg Ganger. Why Can't I Find My Files? New methods for automating attribute assignment. Proceedings of the Ninth Workshop on Hot Topics in Operating systems, USENIX Association, May 2003.

20. P.M. Chen, E.K. Lee, G.A. Gibson, R.H. Katz, and D.A. Patterson, RAID: High Performance and Reliable Secondary Storage, ACM Computing Surveys, pp.145-185, 1994.

21. Website, March, 2005, http://www.ds9a.nl/lvm-howto/

22. Website, May, 2004, http://www.snia.org/

23. T10/1731-D Revision 0, October 2004. SCSI Object-Based Storage Device Commands -2 (OSC-2). Available at http://www.t10.org

24. Website, May, 2004, available at http://sourceforge.net/projects/intel-iscsi/ 